\title{
Practice makes progress: Assessing our robotic skills
}

\author{
Roger F. Valdivieso, MD; Kevin C. Zorn, MD, FACS, FRCSC
}

University of Montreal Hospital Center (CHUM)-Hopital St. Luc, Montreal, QC

See related article on page 430 .

Cite as: Can Urol Assoc J 2013;7(11-12):435-6. http://dx.doi.org/10.5489/cuaj.1782

Published online December 5, 2013.

$\mathrm{T}$ -he use of robotic-assisted laparoscopic procedures is expanding in many surgical disciplines, especially in urology. Technologic peculiarities (3-dimensional vision, wristed instrumentation with 7 degrees of freedom of motion, lack of tremor, a 10-times magnification and a comfortable, seated ergonomic position for the surgeon) have added value to both the surgeon and patient. However, there are still inherent difficulties to the robotic platform; these include the novel use of finger-controls, foot pedals, perspective of the operative field, lack of tactile feedback, and the distancing of surgeon-patient-assistant. The da Vinci Surgical System (Intuitive Surgical, Sunnyvale, CA) requires a learning curve that is not negligible. Many studies have attempted to determine the learning curve and yielded different results. Zorn and colleagues have found that it may take 25 to 50 cases for a fellowship-trained laparoscopic surgeon to overcome the learning curve with respect to operative time and complications. ${ }^{1}$ Similarly, it has been suggested that the oncological learning curve could be as much as 150 cases for a well-experienced open prostatectomy surgeon. ${ }^{2}$ Regardless, it is clear that surgeon experience and surgical volume affect perioperative, oncological and functional outcomes. $^{3-5}$ Therefore, mechanisms and guidelines by which trainee surgeons can attain a sufficient level of skill, without compromising the safety of patients need to be introduced. ${ }^{6}$

Despite the exponential global growth and use of robotic surgery, the educational component to incorporate these skills into clinical practice has been challenging. To improve surgical outcomes and maintain patient safety, the development of robotic surgical skills is migrating towards a simulated, risk-free environment away from the patient. ${ }^{3,7}$ As we are aware, simulators are being used more frequently as

educational tools based on the assumption that repetitive performance of a manual task leads to improvement and learning from failure reduces errors. Simulation's greatest attribute is that it permits trainees to err without jeopardizing patient welfare. The latest robotic simulators provide a computer-derived realistic virtual operative field with tactile feedback on instruments. Currently, there are several daVinci simulators, including Mimic (Mimic Technologies, Seattle WA), ROSS (Simulated Surgical Systems, Williamsville, NY) and the da Vinci Surgical System (dVSS, Intuitive Surgical, Sunnyvale, CA). dVSS is the most popular and integrated training program for acquiring basic skills. The dVSS integrates the previously validated da Vinci Trainer software (Mimic) using the actual daVinci simulator surgeon console as the user interface. Recent studies suggest that this novel simulator demonstrates good content, face and construct validity and can be used for assessing surgeon proficiency with immediate self-assessment feedback. ${ }^{8-10}$ However, there are no standardized robotics training curriculum for residents.

The current article by Foell and colleagues evaluates a simulation based robotic surgery basic skills training curriculum (BSTC) for robotic novices of different surgical disciplines in a Canadian institution. ${ }^{11}$ In the study, a total of 37 subjects completed a 4-week training that included the use of various instructional strategies including the dVSS. Moreover, a group of these participants were involved in a competency-based dVSS training; for these participants, an expert robotic surgeon provided formative feedback upon completion of an exercise and a score of $\geq 80 \%$ was needed to advance through each step of the curriculum. Clearly the use of BSTC significantly improved basic robotic skills regardless of specialty or level of training. It was also found that the competency-based training enhanced the acquisition of basic robotic skills.

The challenge of establishing a training curriculum for robotic surgery is amplified on Canadian soil, as the adoption of robotics is still in its early stages with comparison with the United States. A recent publication demonstrat- 
ed that there is a significant increase in the percentage of minimally invasive radical prostatectomies performed by American urology residents $(11.2 \%-52 \%)$ compared to their Canadian counterparts $(0.74 \%-11.2 \%){ }^{12}$ This discrepancy can be attributed to the different medical systems between the 2 countries. American urologists have rapidly adopted robot-assisted minimally invasive radical prostatectomy, while Canadian urologists have embraced this technology in a more limited fashion, partly because of financial constraints within the Canadian healthcare system. ${ }^{13,14}$ It is under this light that a BSTC, although challenging, is a requirement for a more widespread and safe acceptance of robotic surgery in Canadian institutions.

Competing interests: Dr. Valdivieso declares no competing financial or personal interests. Dr. Zorn is involved with AMS in Greenlight training.

\section{References}

1. Zorn KC, Orvieto MA, Gong EM, et al. Robotic radical prostatectomy learning curve of a fellowship-trained laparoscopic surgeon. J Endourol 2007;21:441-7.

2. Herrell SD, Smith JA Jr. Robotic-assisted laparoscopic prostatectomy: What is the learning curve? Urology 2005;66(5 Suppl):105-7.

3. Ou YC, Yang CR, Wang J, et al. The learning curve for reducing complications of robotic-assisted laparoscopic radical prostatectomy by a single surgeon. BJU Int 2011;108:420-5.
4. Samadi D, Levinson A, Hakimi A, et al. From proficiency to expert, when does the learning curve for roboticassisted prostatectomies plateau? The Columbia University experience. World I Urol 2007;25:105-10.

5. Abdollah F, Budaus L, Sun $M$, et al. Impact of caseload on total hospital charges: a direct comparison between minimally invasive and open radical prostatectomy: A population based study. J Urol 2011;185:855-61.

6. Liberman D, Trinh QD, Jeldres $C$, et al. Training and outcome monitoring in robotic urologic surgery. Nat Rev Urol 2012;9:17-22.

7. Ahlering TE, Skarecky D, Lee D, et al. Successful transfer of open surgical skills to a laparoscopic environment using a robotic interface: Initial experience with laparoscopic radical prostatectomy. J Urol 2003;170:1738-41.

8. Liss MA, Abdelshehid C, Quach S, et al. Validation, correlation, and comparison of the da Vinci trainer() and the daVinci surgical skills simulator using the Mimic software for urologic robotic surgical education. $J$ Endourol 2012;26:1629-34.

9. Hung AJ, Zehnder P, Patil MB, et al. Face, content and construct validity of a novel robotic surgery simulator. J Urol 2011;186:1019-24.

10. Foell K, Furse A, Honey RJA, et al. Multidisciplinary validation study of the da Vinci Skills Simulator: Educational tool and assessment device. J Robotic Surg 2013:1-5.

11. Foell K, Finelli A, Yasufuku K, et al. Robotic surgery basic skills training: Evaluation of a pilot multidisciplinary simulation-based curriculum. Can Urol Assoc J 2013;7:430-4. http://dx.doi.org/10.5489/cuai.222

12. Hoag NA, Mamut A, Afshar $K$, et al. Trends in urology resident exposure to minimally invasive surgery for index procedures: a tale of two countries. J Surg Educ 2012;69:670-5.

13. Fradet $Y$. Arguments against investing widely in robotic prostatectomy in Canada: A wrong focus on tool box rather than surgical expertise. Can Urol Assoc J 2009;3:486-7.

14. Estey EP. Robotic prostatectomy: The new standard of care or a marketing success? Can Urol Assoc J 2009;3:488-90

Correspondence: Dr. Kevin C. Zorn, Assistant Professor of Surgery, Minimally Invasive UrologicalOncologist, Director of Robotic Surgery, University of Montreal Hospital Centre, 235 Rene Levesque Est Suite 301, Montreal, QC H2XIN8 